\title{
Interior Estimates for Ritz-Galerkin Methods
}

\author{
By Joachim A. Nitsche and Alfred H. Schatz
}

\begin{abstract}
Interior a priori error estimates in Sobolev norms are derived from interior RitzGalerkin equations which are common to a class of methods used in approximating solutions of second order elliptic boundary value problems. The estimates are valid for a large class of piecewise polynomial subspaces used in practice, which are defined on both uniform and nonuniform meshes. It is shown that the error in an interior domain $\Omega$ can be estimated with the best order of accuracy that is possible locally for the subspaces used plus the error in a weaker norm over a slightly larger domain which measures the effects from outside of the domain $\Omega$. Additional results are given in the case when the subspaces are defined on a uniform mesh. Applications to specific boundary value problems are given.
\end{abstract}

0. Introduction. There are presently many methods which are available for computing approximate solutions of elliptic boundary value problems which may be classified as Ritz-Galerkin type methods. Many of these methods differ from each other in some respects (for example, in how they treat the boundary conditions) but have much in common in that they have what may be called "interior Ritz-Galerkin equations" which are the same. Here we shall be concerned with finding interior estimates for the rate of convergence for such a class of methods which are consequences of these interior equations. Let us briefly describe, in a special case, the type of question we wish to consider.

Let $\Omega$ be a bounded domain in $\mathbf{R}^{N}$ with boundary $\partial \Omega$ and consider, for simplicity, the problem of finding an approximate solution of a boundary value problem

$$
\begin{aligned}
& \Delta u=f \text { in } \Omega, \\
& A u=g \text { on } \partial \Omega,
\end{aligned}
$$

where $A$ is some boundary operator. Suppose now that we are given a one-parameter family of finite-dimensional subspaces $S^{h}(0<h \leqslant 1)$ of an appropriate Hilbert space in which $u$ lies and that, for each $h$, we have computed an approximate solution $u_{h} \in S^{h}$ to $u$ using some Ritz-Galerkin type method. Here we have in mind, for example, methods such as the "engineer's" finite element method [8], [22], the Aubin-Babuska penalty method [2], [4], the methods of Nitsche [12], [13] or the

Received October 15, 1973.

AMS (MOS) subject classifications (1970). Primary 65N30, 65N 15. 
Lagrange multiplier method of Babuska [3]. Consider now subdomains $\Omega_{0}$ and $\Omega_{1}$ of $\Omega$. with $\Omega_{0} \subset \subset \Omega_{1} \subset \subset \Omega$. We seek estimates for the error $u-u_{h}$ as $h \rightarrow 0$ in various Sobolev norms on $\Omega_{0}$, valid for a large class of such methods. The estimates will implicitly take into consideration possible "pollution" from effects outside of $\Omega_{0}$. These may be, for example, due to the following: (i) the smoothness of the boundary; (ii) the way in which a given method treats the boundary conditions; (iii) the smoothness of the solution outside of say $\Omega_{1}$.

In the special case of Eq. (0.1), our interior equations are as follows: Let $u$ satisfy $(0.1)$ in $\Omega_{1}$ and $u_{h} \in S^{h}$ be given satisfying

$$
\int_{\Omega_{1}} \sum_{i=1}^{N}\left(\frac{\partial u_{n}}{\partial x_{i}}\right)\left(\frac{\partial \varphi}{\partial x_{i}}\right) d x=\int_{\Omega_{1}} f \varphi=\int_{\Omega_{1}}\left(\frac{\partial u}{\partial x_{i}}\right)\left(\frac{\partial \varphi}{\partial x_{i}}\right) d x, \forall \varphi \in \stackrel{\circ}{S}^{h}\left(\Omega_{1}\right),
$$

where $\stackrel{\circ}{S}^{h}\left(\Omega_{1}\right)$ is the subspace of $S^{h}$ consisting of elements whose support is contained in $\Omega_{1}$. For example, we may consider $S^{h}$ to be such that its restriction to $\Omega_{1}$ consists of splines generated by a $B$-spline basis defined on a uniform mesh of size $h$ (cf. [19]). The broad class of triangular elements with maximum size $h$ defined by Bramble and Zlámal [8] (here the triangulation need not be uniform) or Hermite splines on a uniform mesh of size $h$ are other examples of such spaces. The Eq. (0.3) is common to all the methods previously cited.

One type of result we shall prove is the following: Suppose the subspace $S^{h}$ has the following approximability property (and some others shared for example by the subspaces cited above). Let $u \in H^{r}\left(\Omega_{1}\right)$, then there exists a $U_{n} \in S^{h}$ such that

$$
\left\|u-u_{h}\right\|_{0, \Omega_{1}} \leqslant C h^{r}\|u\|_{r, \Omega_{1}}
$$

where $\|\cdot\|_{r,} \Omega_{1}$ is the norm on the Sobolev space $H^{r}\left(\Omega_{1}\right)$ and $H^{0}\left(\Omega_{1}\right)=L_{2}\left(\Omega_{1}\right)$. Suppose further that $u_{h} \in S^{h}$ satisfies (0.3). Then, for any given nonnegative integer $p$, there exists a constant $C$ independent of $h, u$ and $u_{h}$ such that for $h$ sufficiently small

$$
\left\|u-u_{h}\right\|_{0, \Omega_{0}} \leqslant C\left(h^{r}\|u\|_{r, \Omega_{1}}+\left\|u-u_{h}\right\|_{-p, \Omega_{1}}\right) .
$$

Here the negative norm $\|\cdot\|_{-p, \Omega_{1}}$ denotes the norm of dual space of the Sobolev space $\stackrel{\circ}{H}^{p}\left(\Omega_{1}\right)$ (cf. Section 1 ).

In view of (0.4), the estimate (0.5) may be interpreted as follows: If $u \in$ $H^{r}\left(\Omega_{1}\right)$, then the error in the norm of $L_{2}\left(\Omega_{0}\right)$ over any compact subdomain $\Omega_{0}$ of $\Omega_{1}$ may be estimated with the best order of accuracy that the subspace $S^{h}$ can provide over $\Omega_{1}$ plus the error in the much weaker norm of $H^{-p}\left(\Omega_{1}\right)$. We emphasize that, since $(0.3)$ is local in nature, so is the estimate $(0.5)$, in the sense that, when related to boundary value problems, they do not explicitly involve the particular boundary operators, the nature of the boundary, the manner in which the particular method treats the boundary operators and the nature of the solution outside of $\Omega_{1}$. As remarked previously, all of these may have an effect on the rate of 
convergence on $\Omega_{0}$. All of these effects are lumped together in the term $\left\|u-u_{h}\right\|_{-p, \Omega_{1}}$, which must be estimated separately for each particular problem. One way this can be done is to first use the inequality $\|e\|_{p}, \Omega_{1} \leqslant \| e L_{p}, \Omega$. For many methods, the estimate for $\|e\|_{-p, \Omega}$ already exists in the literature and is obtained by using a modification of a duality argument of Nitsche [15]. The significance of the negative norm is that, under some very important circumstances, one can prove high rates of convergence in negative norms with relatively less requirements on the smoothness of. $u$ than one would need, for example, to obtain the same rate of convergence for the error in the $L_{2}$ norm.

Interior estimates for $L_{2}$ projections were given in [17]. Interior estimates for elliptic difference operators were obtained by Thomée and Westergren [20] and Thomé [21]. We would like to thank the organizers of the conference "On the mathematical foundations of the finite element method", University of Maryland, Baltimore, June 1972 for allowing us to present the results given in this paper.

An outline of our paper is as follows. Section 1 contains notations and some preliminary notions. In Section 2, we define the approximating properties of the subspaces $S^{h}$ which we shall need. In Section 3, we introduce the interior equations and, in Section 4, we prove some interior duality estimates. Section 5 contains the first of our results on interior rates of convergence which are valid, for example, for some classes of piecewise polynomial subspaces which may be defined on uniform or nonuniform meshes. In Section 6, we consider subspaces which have certain translation invariant properties (satisfied by the previous examples defined on uniform meshes). This allows us to discuss the interior rate of convergence of difference quotients of $u_{h}$ to derivatives of $u$. In Section 7, we apply the results to specific boundary value problems and to several methods.

This paper is concerned with interior Galerkin equations associated with second order differential operators. The methods easily generalize to corresponding higher order equations.

1. Notation and Preliminaries. All functions considered in this paper will be real valued. Let $R$ be a bounded open set in $\mathbf{R}^{N}$ ( $N$-dimensional Euclidean space).

For $s \geqslant 0$ any real number $H^{s}(R)$ will denote the Sobolev space of order $s$ on $R$, i.e., for $s \geqslant 0$ an integer, $H^{s}(R)$ is the completion of $C^{\infty}(\bar{\Omega})$ under the norm

$$
\|u\|_{s, R}=\left(\sum_{|\alpha| \leqslant s}\left\|D^{\alpha} u\right\|_{0, R}^{2}\right)^{1 / 2},
$$

where $H^{0}(R)=L_{2}(R)$ and $\|u\|_{0, R}^{2}=\int_{R}|u|^{2} d x$. For $k \geqslant 0$, an integer. and $k<s<k+1, H^{s}(R)$ is defined by interpolation between $H^{k}(R)$ and $H^{k+1}(R)$ (cf. [11]). 
For $s \geqslant 0$ an integer, $H^{s}(R)$ will denote the completion of $C_{0}^{\infty}(R)$ under the norm (1.1). For $k \geqslant 0$ an integer and $k<s<k+1, \stackrel{\circ}{H}^{s}(R)$ is defined by interpolation between $\stackrel{\circ}{H}^{k}(R)$ and $H^{k+1}(R)$.

For $s \leqslant 0$ any real number, $\stackrel{H}{H}^{s}(R)$ will denote the completion of $C_{0}^{\infty}(R)$ under the norm

$$
\|u\|_{s, R}=\sup _{v \in \stackrel{\circ}{H}^{-s}(R)}(u, v) /\|v\|_{-s, R}
$$

where $(u, v)=\int_{R} u v d x$.

For $s \leqslant 0$ any real number, $H^{s}(\Omega)$ will denote the completion of $C^{\infty}(R)$ under the norm

$$
\|u\|_{s, R}=\sup _{v \in H^{-s}(R)}(u, v) /\|v\|_{-s, R} .
$$

Let $\Omega_{1}$ be a bounded open set in $\mathbf{R}^{N}$. In what follows, we shall be concerned with bilinear forms of the type

$$
B(u, v)=\int_{\Omega_{1}}\left(\sum_{i, j=1}^{N} a_{i j}(x) D_{i} u D_{j} v+\sum_{i=1}^{N} b_{i}(x)\left(D_{i} u\right) v+c(x) u v\right) d x,
$$

defined on $H^{1}\left(\Omega_{1}\right) \times H^{1}\left(\Omega_{1}\right)$, where, for simplicity, the coefficients $a_{i j}, b_{i}, c$ are assumed to be of class $C^{\infty}\left(\bar{\Omega}_{1}\right)$.

Such forms may be associated (in a nonunique way) with partial differential operators of the form

$$
L u=-\sum_{i, j=1}^{N} a_{i j} D_{i} D_{j} u+\sum_{i=1}^{N} a_{i} D_{i} u+a u=f .
$$

The form $B$ comes about by integrating by parts so that

$$
(L u, v)=B(u, v)
$$

holds for all $u, v \in C_{0}^{\infty}\left(\Omega_{1}\right)$.

In what follows, $C_{1}, C_{2}, \cdots, C_{t}$ will denote constants. We shall frequently omit subscripts and simply write $C$ for a constant which is not necessarily the same in any two places. $C(G, p)$ means, for example, that the constant $C$ depends on the known parameters $G$ and $p$.

We shall not require that $B(u, v)$ be symmetric. We define the adjoint $B^{*}$ of $B$ to be

$$
B^{*}(u, v)=B(v, u)
$$

The following regularity assumption will be made concerning $B(u, v)$ :

R1. There exists a constant $d_{1}>0$ such that, if $G \subset \subset \Omega_{1}$ is any (open) sphere with $\operatorname{diam}(G) \leqslant d_{1}$, then $B(u, v)$ is coercive over $\stackrel{\circ}{H}^{1}(G)$, i.e., there exists a constant $C_{1}$ depending only on $d_{1}$ such that

$$
\left(C_{1}\right)^{2}\|u\|_{1, G}^{2} \leqslant B(u, u) \leqslant\left(C_{1}\right)^{-2}\|u\|_{1, G}^{2} \text { for all } u \in \stackrel{\circ}{H}^{1}(G) \text {. }
$$

Remark 1.1. It follows immediately from (1.5) that $B^{*}$ satisfies $\mathrm{R} 1$. 
Remark 1.2. Assumption $\mathrm{R} 1$ is always satisfied if $d_{1}$ is sufficiently small, provided $B(u, v)$ is uniformly elliptic on $\Omega_{1}$, i.e., there exists a constant $C>0$ such that for all $x \in \bar{\Omega}_{1}$ and all real vectors

$$
\zeta=\left(\zeta_{1}, \cdots, \zeta_{N}\right) \neq 0,\left|\sum_{j=1}^{N} \sum_{i=1}^{N} a_{i j}(x) \zeta_{i} \zeta_{j}\right| \geqslant C \sum_{i=1}^{N} \zeta_{i}^{2} .
$$

For then Garding's inequality holds in $\Omega_{1}$, i.e., there exist positive constants $C_{2}$ and $C_{3}$ such that

$$
C_{2}\|u\|_{1, \Omega_{1}}^{2}-C_{3}\|u\|_{0, \Omega_{1}}^{2} \leqslant B(u, u) \text { for all } u \in \stackrel{\circ}{H}^{1}\left(\Omega_{1}\right) .
$$

Now, Poincare's inequality states that

$$
\|u\|_{0, G} \leqslant \operatorname{diam}(G)\|u\|_{1, G} \text { for all } u \in \stackrel{\circ}{H}^{1}(G) .
$$

The inequality (1.7) follows immediately from the last two inequalities by taking $\operatorname{diam}(G)<d_{1}$ to be sufficiently small.

We shall find the following lemma useful later on.

Lemma 1.1. (Cf. e.g. [1], [5] .) Let $G \subset \subset \Omega_{1}$ be a sphere with diam $(G) \leqslant d_{1}$. Suppose that $f \in H^{s}(G), s \geqslant 0$, then there exist uniquely determined functions $u$ and $v$ belonging to $H^{s+2}(G) \cap \stackrel{\circ}{H}^{1}(G)$ satisfying

$$
B(u, \psi)=B^{*}(v, \psi)=(f, \psi) \text { for all } \psi \in \stackrel{\circ}{H}^{1}(G)
$$

Furthermore, there exists a constant $C$, which depends on $s$ but is independent of $f$ and $G$, such that

$$
\|u\|_{s+2, G} \leqslant C\|f\|_{s, G}, \quad\|v\|_{s+2, G} \leqslant C\|f\|_{s, G} .
$$

2. Finite-Dimensional Subspaces of $H^{1}\left(\Omega_{1}\right)$. Let $\Omega_{1} \subset \subset \mathbf{R}^{N}$ be an open set. In this section, we shall define a class of finite-dimensional subspaces of $H^{1}\left(\Omega_{1}\right)$ which have properties that are shared by many finite-dimensional subspaces used in practice to approximate solutions of partial differential equations. We shall first state the approximation properties which we shall need and then give some examples.

Let $h$ be a parameter and $k$ and $r$ given integers with $1 \leqslant k \leqslant r . \quad S_{k,}^{h} \Omega_{1}$ ) will denote a one-parameter family of finite-dimensional subspaces of $H^{k}\left(\Omega_{1}\right)$. For $\Omega_{0} \subseteq \Omega_{1}$, we define

$$
\dot{S}_{k, r}^{h}\left(\Omega_{0}\right)=\left\{\varphi \in S_{k, r}^{h}\left(\Omega_{1}\right) \mid \operatorname{supp} \varphi \subseteq \bar{\Omega}_{0}\right\} .
$$

Let $G_{0}$ and $G$, with $G_{0} \subset \subset G \subset \subset \Omega_{1}$, be arbitrary but fixed concentric spheres. We shall make the following approximability assumptions concerning $S_{k, r}^{h}\left(\Omega_{1}\right)$ :

There exists an $h_{0} \leqslant 1$, depending in general on $G_{0}$ and $G$, such that for all $h \in\left(0, h_{0}\right]:$

A.1. (i) For each $u \in H^{l}(G)$, there exists an $\eta \in S_{k, r}^{h}\left(\Omega_{1}\right)$ such that for any $0 \leqslant s \leqslant k, s \leqslant l \leqslant r$,

$$
\|u-\eta\|_{s, G} \leqslant C h^{l-s}\|u\|_{l, G}
$$


(ii) Furthermore, if $u \in \stackrel{\circ}{H}^{l}\left(G_{0}\right)$, then $\eta \in \stackrel{\circ}{S}_{k, r}^{h}(G)$.

REMARK. If $\omega \in C_{0}^{\infty}\left(G_{0}\right)$ and $u \in H^{l}(G)$, then $\omega u \in \stackrel{\circ}{H}^{l}\left(G_{0}\right)$ and it follows from (ii) that $\eta \in \stackrel{\circ}{S}_{k, r}^{h}(G)$ and

$$
\|\omega u-\eta\|_{s, G} \leqslant C h^{l-s}\|u\|_{l, G},
$$

where $C=C\left(G_{0}, G, \omega\right)$.

A.2. Let $\omega \in C_{0}^{\infty}\left(G_{0}\right)$ and $u_{h} \in S_{k, r}^{h}\left(\Omega_{1}\right)$, then there exists an $\eta \in \stackrel{\circ}{S}_{k, r}^{h}(G)$ such that

$$
\left\|\omega u_{h}-\eta\right\|_{1, G} \leqslant C h\left\|u_{h}\right\|_{1, G}
$$

where $C=C\left(G_{0}, G, \omega\right)$.

A.3. For' each $h \in\left(0, h_{0}\right]$, there exists a set $G_{h}, G_{0} \subset \subset G_{h} \subset \subset G$ such that, if $0 \leqslant \nu \leqslant s \leqslant k$, then for all $\varphi \in S_{k, r}^{h}\left(\Omega_{1}\right)$

$$
\|\varphi\|_{s, G_{h}} \leqslant C h^{\nu-s}\|\varphi\|_{\nu, G_{h}} .
$$

Remark. If Green's formula is valid on $G_{h}$ and $p \geqslant 1$ is an integer, then it can be shown, as a consequence of (2.4), that

where $C=C\left(G_{0}, G\right)$.

$$
\|\varphi\|_{0, G_{h}} \leqslant C h^{-p}\|\varphi\|_{p, G_{h}}
$$

We shall now give some examples of subspaces which satisfy A.1, A.2, and A.3.

Example 1. Let $S_{k, r}^{n}\left(\Omega_{1}\right)$ denote the restriction to $\Omega_{1}$ of Hermite splines defined on a uniform mesh with sides of length $h$. Here $k=m, r=2 m, m=1,2$, $\cdots$, etc. For $m=2$, these are the piecewise cubic polynomials which are of class $C^{\mathbf{1}}$.

Example 2. Consider $\mathbf{R}^{2}$ and let $S_{k, r}^{h}\left(\Omega_{1}\right)$ be the restrictions to $\Omega_{1}$ of the triangular elements of Bramble-Zlámal [8]. Briefly, these are piecewise polynomials in two variables of order $4 m+1(m=0,1, \cdots)$ defined on a regular triangulation (i.e., the smallest interior angle of all the triangles is uniformly bounded away from zero) whose length of the largest side of any triangle is less than or equal to $h$. We note here that these triangulations are generally not uniform. Here, we may take $k=m+1$ and $r=4 m+2(m=0,1, \cdots)$.

Example 3. Let $S_{k, r}^{h}\left(\Omega_{1}\right)$ be the restriction to $\Omega_{1}$ of splines generated by a $B$-spline basis [19], defined on a uniform mesh in $\mathbf{R}^{N}$ with sides of length $h$. These are tensor products of one-dimensional piecewise polynomials of order $m-1$ which are globally $C^{m-2}(m=2,3, \cdots)$. Here, $k=m-1, r=m \quad(m=2,3, \cdots)$.

For these examples, Property A.1 is well known. Property A.3 follows easily from [14]. Property A.2 is more delicate than Property A.1. We shall verify it here in the case of Example 2.

Let $G_{0}, G, \omega$ and $u_{h}$ be as in A.2, and let $G$ be covered by a regular triangulation on which the Bramble-Zlámal elements are defined. Let us number the triangles by $T_{i}$. Then it follows from (15) of [8] that 
where

$$
\left\|\omega u_{h}-\eta\right\|_{1, G}^{2} \leqslant C h^{2(4 m+1)} \sum_{i}\left|\omega u_{h}\right|_{4 m+2, T_{i}}^{2}
$$

$$
|u|_{4 m+2, T_{i}}^{2}=\sum_{|\alpha|=4 m+2}\left\|D^{\alpha} u\right\|_{0, T_{i}}^{2}
$$

and the sum in (2.6) is taken over those triangles $T_{i}$ such that their union contains the support of $\omega$. Certainly, this may be chosen so that $\bigcup_{i} T_{i} \subset G$ for $h$ sufficiently small. Now, using Leibnitz's rule on each of the triangles $T_{i}$, noticing that $D^{\alpha} u_{h}=0$ for ${ }^{\circ}|\alpha|=4 m+2$ (since $u_{h}$ is a polynomial of order $4 m+1$ in two variables), we obtain

$$
\left\|\omega u_{h}-\eta\right\|_{1, G}^{2} \leqslant C h^{2(4 m+1)} \sum_{i}\left\|u_{h}\right\|_{4 m+1, T_{i}}^{2}
$$

where $C$ in general depends on $\omega$. Since $u_{h}$ is a polynomial and the triangulation is regular, we have

$$
\left\|u_{h}\right\|_{4 m+1, T_{i}} \leqslant C h^{-4 m}\left\|u_{h}\right\|_{1, T_{i}}
$$

where $C$ is independent of $u_{h}$ and $T_{i}$. Hence,

$$
\left\|\omega u_{h}-\eta\right\|_{1, G}^{2} \leqslant C h^{2} \sum_{i}\left\|u_{h}\right\|_{1, T_{i}}^{2} \leqslant C h^{2}\left\|u_{h}\right\|_{1, G}^{2}
$$

which is precisely (2.3).

We remark that the proof of Property A.2 in the case of Hermite splines (Example 1) follows in a similar manner using the results of Bramble-Hilbert in [6], where an estimate is obtained analogous to (2.6). Weamake essential use of the fact that only those derivatives which annihilate the Hermite splines occur on the right-hand side.

3. Interior Equations. Let $B(u, v)$ be defined by (1.3) and $u \in H^{1}\left(\Omega_{1}\right)$. We shall be primarily interested in deriving error estimates for $u-u_{h}$, where $u_{h} \in$ $S_{k, r}^{h}\left(\Omega_{1}\right)$ satisfies

$$
B\left(u-u_{h}, \varphi\right)=0 \quad \forall \varphi \in \stackrel{\circ}{S}_{k, r}^{h}\left(\Omega_{1}\right) .
$$

Let us note that, in view of (1.4) and (1.5), (3.1) may be rewritten as

$$
B\left(u_{h}, \varphi\right)=(L u, \varphi)=(f, \varphi) \quad \forall \varphi \in \stackrel{\circ}{S}_{k, r}^{h}\left(\Omega_{1}\right) .
$$

An interesting special case occurs when $L u=0$ or $B(u, v)=0 \quad \forall v \in H^{1}\left(\Omega_{1}\right)$, for then $u_{h} \in S_{k, r}^{h}\left(\Omega_{1}\right)$ satisfies

$$
B\left(u_{h}, \varphi\right)=0 \quad \forall \varphi \in \stackrel{\circ}{S}_{k, r}^{n}\left(\Omega_{1}\right) .
$$

Here, $u_{n}$ may be thought of as a discrete analogue (relative to the subspace $S_{k, r}^{h}\left(\Omega_{1}\right)$ ) of a weak solution of $L u=0$ in $\Omega_{1}$. Such $u_{h}$ will play a central role in deriving error estimates.

In obtaining error estimates for difference quotients for certain classes of sub- 
spaces, it will be convenient for us to consider $u_{h}$ satisfying a more general form of (2.1); namely

$$
B(e, \varphi)=B\left(u-u_{h}, \varphi\right)=A_{e}(\varphi) \forall \varphi \in \stackrel{\circ}{S}_{k, r}^{n}\left(\Omega_{1}\right)
$$

In general, $A_{v}(\omega)$ is, for each $v \in H^{1}\left(\Omega_{1}\right)$, a bounded linear functional defined for $\omega \in \stackrel{\circ}{H^{s}}\left(\Omega_{1}\right)$ for any $-1 \leqslant s$.

For any open set $G \subseteq \Omega_{1}$ and $-1 \leqslant s$, an integer, we define

$$
\left\|A_{e}\right\|_{s, G}=\sup _{v \in H^{s+2}(G)}\left|A_{e}(v)\right| /\|v\|_{s+2, G} .
$$

Here, we are essentially defining the norms on $A_{e}$ by duality with respect to the $H^{1}(G)$ norm.

Let us note that, if $G_{0} \subseteq G_{1}$, then

and, if $-1 \leqslant s_{1} \leqslant s_{2}$, then

$$
\left\|A_{e}\right\|_{s, G_{0}} \leqslant\left\|A_{e}\right\|_{-s, G_{1}},
$$

$$
\left\|A_{e}\right\|_{-s_{2}, G} \leqslant\left\|A_{e}\right\|_{-_{1}, G}
$$

4. Interior Duality Estimates. For the remainder of this section, we shall assume that R.1, A.1, A.2 and A.3 hold and that $G_{0} \subset \subset G$ are concentric spheres with $\operatorname{diam}(G) \leqslant d_{1} \quad$ (as in R.1) and $G \subset \subset \Omega_{1}$. We shall now discuss the properties of the error $e=u-u_{h}$ satisfying $B(e, \varphi)=A_{e}(\varphi)$ for all $\varphi \in \stackrel{\circ}{S}_{k, r}^{h}(G)$.

The main result in this section is the following:

Lemma 4.1. Let $u \in H^{1}\left(\Omega_{1}\right), u_{h} \in S_{k, r}^{h}\left(\Omega_{1}\right)$ with $1 \leqslant k<r$ and let $p \geqslant 0$ be a fixed but arbitrary integer. Let $G_{0} \subset \subset G$ be as above and suppose that $e=$ $u-u_{h}$ satisfies

$$
B\left(u-u_{h}, \varphi\right)=A_{e}(\varphi), \quad \forall \varphi \in \stackrel{\circ}{S}_{k, r}^{h}\left(\Omega_{1}\right)
$$

Then

$$
\|e\|_{0, G} \leqslant C\left(h\|e\|_{1, G}+\|e\|_{-p, G}+h\left\|A_{e}\right\|_{1, G}+\left\|A_{e}\right\|_{0, G}\right),
$$

where $C=C\left(G_{0}, G, p, a_{i j}, b_{i}, c\right)$.

In order to prove Lemma 4.1, we shall use the following:

Lemma 4.2. Let $s \geqslant 0$ be an integer. Then, for $\gamma=\min (s+1, r-1)$,

$$
\|e\|_{s, G} \leqslant C\left(h^{\gamma}\|e\|_{1, G}+\|e\|_{-s-1, G}+h^{\gamma}\left\|A_{e}\right\|_{1, G}+\left\|A_{e}\right\|_{-s, G}\right)
$$

where $C=C\left(s, G_{0}, G, a_{i j}, b_{i}, c\right)$.

Proof of Lemma 4.1. Let us first show that (4.2) follows from (4.3). Let $G_{0} \subset \subset G_{1} \subset \subset \cdots \subset \subset G_{p}=G$ be concentric spheres. We have from (4.3), with $s=0$ and $\gamma=1$, that

$$
\|e\|_{0, G_{0}} \leqslant C\left(h\|e\|_{1, G_{1}}+\|e\|_{1_{1}, G_{1}}+h\left\|A_{e}\right\|_{1, G_{1}}+\left\|A_{e}\right\|_{0, G_{1}}\right) .
$$


We now reapply (4.3) to estimate $\|e\|_{-1, G}$. Since $h \leqslant 1$, we have that

$$
\|e\|_{0, G_{0}} \leqslant C\left(h\|e\|_{1, G_{2}}+\|e\|_{-2, G_{2}}+h\left\|A_{e}\right\|_{1, G_{2}}+\left\|A_{e}\right\|_{0, G_{2}}\right)
$$

where we used (3.6) and (3.7). Continuing in this fashion, the desired result (4.2) is easily obtained.

Proof of Lemma 4.2. Let $G_{0} \subset \subset G^{\prime} \subset \subset G$ be concentric spheres and let $\omega \in C_{0}^{\infty}\left(G^{\prime}\right)$ with $\omega \equiv 1$ on $G_{0}$. Then, for $s \geqslant 0$, we have that

$$
\left\|e L_{s, G_{0}} \leqslant\right\| \omega e\left\|_{-s, G}=\sup _{f \in \stackrel{\circ}{H}^{s}(G)}(\omega e, f) /\right\| f \|_{s, G} .
$$

Now, we $\in \stackrel{\circ}{H}^{1}(G)$ and it follows from Lemma 1.1 that, for each $f \in H^{s}(G)$, there exists a unique $v \in H^{s+2}(G) \cap \stackrel{\circ}{H}^{1}(G)$ such that $(\eta, f)=B(\eta, v)$ for all $\eta \in \stackrel{\circ}{H}^{1}(G)$ with $\|v\|_{s+2, G} \leqslant C\|f\|_{s, G}$. Hence

$$
\|e\|_{s, G_{0}} \leqslant C \sup _{v \in H^{s+2}(G)} B(\omega e, v) /\|v\|_{s+2, G} .
$$

Let us now examine $B(\omega e, v)$ in detail. We have

$$
\begin{aligned}
B(\omega e, v)= & \int_{G} \sum_{i=1}^{N} \sum_{j=1}^{N} a_{i j} D_{i}(\omega e) D_{j} v d x \\
& +\int_{G} \sum_{i=1}^{N} b_{i}\left(D_{i}(\omega e)\right) v d x+\int_{G} c \omega e v d x
\end{aligned}
$$

Integration by parts yields

$$
B(\omega e, v)=B(e, \omega v)
$$

$$
\begin{aligned}
& +\int_{G} \sum_{j=1}^{N} \sum_{i=1}^{N} e\left[D_{i}\left(a_{i j}\left(D_{j} \omega\right) v\right)+a_{i j} D_{i} \omega D_{j} v+b_{i}\left(D_{i} \omega\right) v\right] d x \\
= & B(e, \omega v)+I .
\end{aligned}
$$

We shall now estimate these terms. Since $e$ satisfies (4.1), we have, for any $\psi \in \stackrel{\circ}{S}_{k, r}^{h}(G)$,

$$
|B(e, \omega v)| \leqslant|B(e, \omega v-\psi)|+\left|A_{e}(\omega v-\psi)\right|+\left|A_{e}(\omega v)\right| .
$$

Choosing $\psi$ satisfying (2.2), we obtain, using (3.5), that

$$
|B(e, \omega v)| \leqslant C\left(h^{\gamma}\|e\|_{1, G}+h^{\gamma}\left\|A_{e}\right\|_{1, G}+\left\|A_{e}\right\|_{s, G}\right)\|v\|_{s+2, G},
$$
where $\gamma=\min (r-1, s+1)$.

Since $D_{i} \omega \in C_{0}^{\infty}\left(G^{\prime}\right)$, it follows that

$$
|I| \leqslant C\|e\|_{s-1, G}\|v\|_{s+2, G} .
$$

The inequality (4.3) now follows from (4.8), (4.7), (4.6) and (4.4).

5. Interior Error Estimates. We now turn to error estimates for solutions of (3.1). We shall first state our results.

THEOREM 5.1. Let $\Omega_{0} \subset \subset \Omega_{1} \subset \subset \mathbf{R}^{N}, u \in H^{l}\left(\Omega_{1}\right), u_{h} \in S_{k, r}^{h}\left(\Omega_{1}\right)$, where $1 \leqslant k<r$ and $p$ is a nonnegative integer, arbitrary but fixed. Suppose that R.1, 
A.1, A.2 and A.3 are satisfied. There exists $a \quad 0<h_{1} \leqslant 1$ such that, if $e=u-u_{h}$ satisfies (3.1), then for all $h \in\left(0, h_{1}\right]$

(i) If $s=0,1$ and $1 \leqslant l \leqslant r$,

$$
\|e\|_{s, \Omega_{0}} \leqslant C\left(h^{l-s}\|u\|_{l, \Omega_{1}}+\|e\|_{-p, \Omega_{1}}\right) \text {. }
$$

(ii) If $2 \leqslant s \leqslant l \leqslant r, s \leqslant k<r$, then

$$
\|e\|_{s, \Omega_{0}} \leqslant C\left(h^{l-s}\|u\|_{l, \Omega_{1}}+h^{1-s}\|e\|_{p, \Omega_{1}}\right)
$$

where $C=C\left(p, \Omega_{0}, \Omega_{1}, a_{i j}, b_{i}, c\right)$.

We note that if $B(u, v)=0 \quad \forall v \in \stackrel{\circ}{H}^{1}\left(\Omega_{1}\right)$, then $u_{n}$ satisfies

$$
B\left(u_{n}, \varphi\right)=0 \quad \forall \varphi \in \stackrel{\circ}{S}_{k, r}^{h}\left(\Omega_{1}\right) .
$$

Using Theorem 5.1, we may obtain estimates for both $u$ and $u_{h}$. In particular, for $u_{h}$ we are at liberty to set $u \equiv 0$ in Theorem 5.1 and we have

$$
\left\|u_{h}\right\|_{1, \Omega_{0}} \leqslant C\left\|u_{h}\right\|_{-p, \Omega_{1}} .
$$

If $u-u_{h}$ satisfies (3.4) we have:

THEOREM 5.2. Suppose that the conditions of Theorem (5.1) are satisfied except that $e=u-u_{n}$ satisfies (3.4); then instead of (5.1) we have

$$
\|e\|_{0, \Omega_{0}} \leqslant C\left(h^{l}\|u\|_{l, \Omega_{1}}+\|e\|_{-p, \Omega_{1}}+h\left\|A_{e}\right\|_{1, \Omega_{1}}+\left\|A_{e}\right\|_{0, \Omega_{1}}\right)
$$

and

$$
\|e\|_{1, \Omega_{0}} \leqslant C\left(h^{l-1}\|u\|_{l, \Omega_{1}}+\|e\|_{-p, \Omega_{1}}+\left\|A_{e}\right\|_{1, \Omega_{1}}\right),
$$

where $C=C\left(p, \Omega_{0}, \Omega_{1}, a_{i j}, b_{i}, c\right)$.

We shall first prove a local version (Lemma 5.2) of the estimate (5.4). For the remainder of this section, we shall assume that R.1, A.1, A.2 and A.3 hold.

LEMma 5.1. Suppose that $u_{h} \in S_{k, r}^{h}\left(\Omega_{1}\right)$ satisfies (5.3) and that $G_{0} \subset \subset$ $G \subset \subset \Omega_{1}$ are concentric spheres with $\operatorname{diam}(G)<d_{1}$. Let $p$ be a nonnegative integer arbitrary but fixed; then, for $h$ sufficiently small,

$$
\left\|u_{h}\right\|_{1, G} \leqslant C\left(h\left\|u_{h}\right\|_{1, G}+\left\|u_{h}\right\|_{p, G}\right)
$$

where $C=C\left(p, G_{0}, G, a_{i j}, b_{i}, c\right)$.

Proof. For any given $v \in \stackrel{\circ}{H}^{1}(G)$, let $P v \in \stackrel{\circ}{S}_{k, r}^{h}(G)$ and $P^{*} v \in \stackrel{\circ}{S}_{k, r}^{h}(G)$ be defined as the solutions of the equations

$$
\begin{aligned}
& B(v-P v, \varphi)=0, \quad \forall \varphi \in \stackrel{\circ}{S}_{k, r}^{h}(G), \\
& B\left(\chi, v-P^{*} v\right)=0, \quad \forall \chi \in \stackrel{\circ}{S}_{k, r}^{h}(G) .
\end{aligned}
$$

It follows from (1.7) that $P v$ and $P^{*} v$ exist and are unique and furthermore 


$$
\begin{aligned}
\|P v\|_{1, G} & \leqslant C \sqrt{B(P v, P v)} \leqslant C \sup _{\substack{\varphi \in \dot{S}_{k, r}^{h}(G) \\
k}} B(P v, \varphi) /\|\varphi\|_{1, G} \\
& \leqslant C \sup _{\varphi \in \stackrel{S}{S}_{k, r}^{h}(G)} B(v, \varphi) /\|\varphi\|_{1, G} \leqslant\|v\|_{1, G} .
\end{aligned}
$$

In a similar fashion, we obtain

$$
\left\|P^{*} v\right\|_{1, G} \leqslant C\|v\|_{1, G}
$$

Let $G_{0} \subset \subset G^{\prime} \subset \subset G$ be concentric spheres, $\omega \in C_{0}^{\infty}\left(G^{\prime}\right)$ with $\omega \equiv 1$ on $G_{0}$ and set $\tilde{u}_{h}=\omega u_{h}$. We have

$$
\left\|u_{h}\right\|_{1, G} \leqslant\left\|\tilde{u}_{h}\right\|_{1, G} \leqslant\left\|\tilde{u}_{h}-P \tilde{u}_{h}\right\|_{1, G}+\left\|P \tilde{u}_{h}\right\|_{1, G} .
$$

We shall estimate these terms separately. Since $P \tilde{u}_{h} \in \stackrel{\circ}{h}_{k, r}(G)$ satisfies (5.8) with $v=\tilde{u}_{h}$, it follows from (1.7) that

$$
\left\|\tilde{u}_{h}-P \tilde{u}_{h}\right\|_{1, G} \leqslant C\left\|\tilde{u}_{h}-\eta\right\|_{1, G} \text { for any } \eta \in \stackrel{\circ}{S}_{k, r}^{h}(G) \text {. }
$$

In view of (2.3), we have

$$
\left\|\tilde{u}_{h}-P \tilde{u}_{h}\right\|_{1, G} \leqslant C h\left\|u_{h}\right\|_{1, G} .
$$

Now let us consider the second term on the right-hand side of (5.12). For $P \tilde{u}_{h} \neq 0$, we have

$$
\left\|P \tilde{u}_{h}\right\|_{1, G} \leqslant C B\left(P \tilde{u}_{h}, \psi\right)=C B\left(\tilde{u}_{h}, \psi\right),
$$

where $\psi=P \tilde{u}_{n} /\left\|P \tilde{u}_{n}\right\|_{1, G}$ and therefore $\|\psi\|_{1, G}=1$. It follows from (4.6) that

$$
\begin{aligned}
B\left(\tilde{u}_{h}, \psi\right)= & B\left(u_{h}, \widetilde{\psi}\right) \\
& +\int_{G} \sum_{i, j=1}^{N} u_{h}\left[D_{i}\left(a_{i j}\left(D_{j} \omega\right) \psi\right)+a_{i j} D_{i} \omega D_{j} \psi+b_{i}\left(D_{i} \omega\right) \psi\right] d x \\
= & B\left(u_{h}, \widetilde{\psi}\right)+I .
\end{aligned}
$$

Since $u_{h}$ satisfies (5.3) and $\operatorname{supp}(\omega) \subseteq G^{\prime}$, we have

$$
B\left(\widetilde{u}_{h}, \psi\right)=B\left(u_{h}, \widetilde{\psi}-\eta\right)+I \text { for any } \eta \in \stackrel{\circ}{S}_{k, r}^{n}(G) .
$$

In view of (2.3) and the definition of $I$, it follows that

$$
B\left(\tilde{u}_{h}, \psi\right) \leqslant C\left(h\left\|u_{h}\right\|_{1, G}+\left\|u_{h}\right\|_{0, G^{\prime}}\right) .
$$

Applying Lemma 4.1 with $e=u_{h}$ and $A_{e}=0$ and $G^{\prime}$ in place of $G_{0}$, we obtain

$$
B\left(\tilde{u}_{h}, \psi\right) \leqslant C\left(h\left\|u_{h}\right\|_{1, G}+\left\|u_{h}\right\|_{-p, G}\right) .
$$

The estimate (5.7) now easily follows from (5.12), (5.13), (5.14) and (5.15), which completes the proof.

Lemma 5.2. Suppose the conditions of Lemma 5.1 are satisfied; then

$$
\left\|u_{h}\right\|_{1, G_{0}} \leqslant C\left\|u_{h}\right\|_{-p, G},
$$

where $C=C\left(p, G_{0}, G, a_{i j}, b_{i}, c\right)$.

Proof. Let $G_{0} \subset \subset G_{1} \subset \subset \cdots \subset \subset G_{p+2}=G$ be concentric spheres. Lemma 
5.1 applies to each pair $G_{j} \subset \subset G_{j+1}$ (with possibly different constants $C_{j}$ ). We have from (5.5) that

$$
\left\|u_{h}\right\|_{1, G_{j}} \leqslant C_{j}\left(h\left\|u_{h}\right\|_{1, G_{j+1}}+\left\|u_{h}\right\|_{-p, G_{j+1}}\right) .
$$

Starting with $j=0$ and iterating $p+1$ times, we obtain

$$
\left\|u_{h}\right\|_{1, G_{0}} \leqslant C\left(h^{p+1}\left\|u_{h}\right\|_{1, G_{p+1}}+\left\|u_{h}\right\|_{-p, G_{p+1}}\right) .
$$

Now, let $G_{h}, G_{p+1} \subset \subset G_{h} \subset \subset G_{p+2}=G$, be as in A.3, then it follows from (2.4) and (2.5) that

$$
h^{p+1}\left\|u_{h}\right\|_{1, G} \leqslant h^{p+1}\left\|u_{h}\right\|_{1, G_{h}} \leqslant C\left\|u_{h}\right\|_{p, G_{h}} \leqslant C\left\|u_{h}\right\|_{-p, G} .
$$

The inequality (5.16) now follows immediately from (5.17) and (5.18).

We shall now prove a local version of Theorems 5.1 and 5.2.

LEMmA 5.3. Suppose the conditions of Theorem 5.2 are satisfied, then (5.1), (5.2), (5.5) and (5.6) hold with $\Omega_{0}$ and $\Omega$ replaced by $G_{0}$ and $G$, concentric spheres satisfying the conditions of Lemma 5.1.

Proof. Let $G_{0} \subset \subset G_{0}^{\prime} \subset \subset G^{\prime} \subset \subset G \subset \subset \Omega_{1}$ and $\omega=1$ on $G_{0}^{\prime}, \omega \in C_{0}^{\infty}\left(G^{\prime}\right)$ and set $\tilde{u}=\omega u$. Let $T \tilde{u} \in \stackrel{\circ}{S}_{k, r}^{h}(G)$ be the unique solution of

$$
B(\tilde{u}-T \tilde{u}, \varphi)=A_{e}(\varphi) \forall \varphi \in \stackrel{\circ}{S}_{k, r}^{h}(G) .
$$

Now

$$
\begin{aligned}
\|\tilde{u}-T \tilde{u}\|_{1, G} & \leqslant C \sup _{v \in H^{1}(G)} B(\tilde{u}-T \tilde{u}, v) /\|v\|_{1, G} \\
& \leqslant C \sup _{v \in \stackrel{O}{H}^{1}(G)} \frac{B\left(\tilde{u}-T \tilde{u}, v-P^{*} v\right)}{\|v\|_{1, G}}+\frac{A_{e}\left(P^{*} v\right)}{\|v\|_{1, G}},
\end{aligned}
$$

where $P^{*} v \in \stackrel{\circ}{S}_{k, r}^{h}(G)$ satisfies (5.9). Hence, for any $\eta \in \stackrel{\circ}{S}_{k, r}^{h}(G)$,

$$
B\left(\tilde{u}-T \tilde{u}, v-P^{*} v\right)=B\left(\tilde{u}-\eta, v-P^{*} v\right) \leqslant C\|\tilde{u}-\eta\|_{1, G}\|v\|_{1, G},
$$

where we have used (5.11). Also

$$
A_{e}\left(P^{*} v\right) \leqslant\left\|A_{e}\right\|_{1, G}\left\|P^{*} v\right\|_{1, G} \leqslant\left\|A_{e}\right\|_{1, G}\|v\|_{1, G} .
$$

It follows now from (5.20), (5.21), (5.22) and (2.1) that

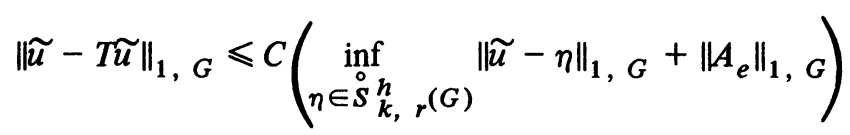

for $1 \leqslant l \leqslant r$.

$$
\leqslant C\left(h^{l-1}\|u\|_{l, G}+\left\|A_{e}\right\|_{1, G}\right)
$$

Let us now estimate $\left\|u-u_{n}\right\|_{1}, G_{0}$.

$$
\left\|u-u_{h}\right\|_{1, G_{0}} \leqslant\|u-T \tilde{u}\|_{1, G_{0}}+\left\|T \tilde{u}-u_{h}\right\|_{1}, G_{0} .
$$

Certainly, Eqs. (3.4) and (5.19) hold for all $\varphi \in \stackrel{\circ}{S}_{k . r}^{h}\left(G_{0}^{\prime}\right)$. Subtracting these 
two equations, we have

$$
B\left(u_{h}-T \tilde{u}, \varphi\right)=0 \quad \forall \varphi \in \stackrel{\circ}{S}_{k, r}^{h}\left(G_{0}^{\prime}\right),
$$

and we may apply Lemma 5.2 to $u_{h}-T \tilde{u}$, with $G_{0}$ and $G$ replaced by $G_{0}$ and $G_{0}^{\prime}$, respectively. It follows then from (5.16) that

$$
\begin{aligned}
\left\|u_{h}-T \tilde{u}\right\|_{1, G} & \leqslant C\left\|u_{h}-T \tilde{u}\right\|_{-p, G}^{\prime} \\
& \leqslant C\left(\left\|u-u_{h}\right\|_{p, G_{0}^{\prime}}+\|\tilde{u}-T \tilde{u}\|_{-p, G_{0}^{\prime}}\right) \\
& \leqslant C\left(\|e\|_{-p, G}+\|\tilde{u}-T \tilde{u}\|_{1, G}\right) .
\end{aligned}
$$

Hence, (5.24) becomes

$$
\left\|u-u_{h}\right\|_{1, G_{0}} \leqslant C\left(\|\tilde{u}-T \tilde{u}\|_{1, G}+\|e\|_{-p, G}\right)
$$

and, applying the estimate (5.23), we have

$$
\|e\|_{1, G} \leqslant C\left(h^{l-1}\|u\|_{l, G}+\|e\|_{-p, G}+\left\|A_{e}\right\|_{1, G}\right)
$$

which proves a local analogue of (5.6) and hence of (5.1) (with $s=1$ by simply taking $A_{e}=0$ ). Let us now prove the local analogues of (5.1) (with $s=0$ ) and (5.5). We first apply Lemma 4.1 to the spheres $G_{0}$ and $G^{\prime}$ and obtain

$$
\|e\|_{0, G_{0}} \leqslant C\left(h\|e\|_{1, G^{\prime}}+\|e\|_{p, G^{\prime}}+h\left\|A_{e}\right\|_{1, G^{\prime}}+\left\|A_{e}\right\|_{0, G^{\prime}}\right) .
$$

Applying the estimate (5.26) to (5.27), with $G_{0}$ and $G$ replaced by $G^{\prime}$ and $G$, respectively, we obtain the desired result

$$
\|e\|_{0, G} \leqslant C\left(h^{l}\|u\|_{l, G}+\|e\|_{p, G}+h\left\|A_{e}\right\|_{1, G}+\left\|A_{e}\right\|_{0, G}\right) .
$$

We now turn to the proof of a local version of (5.2). Let $2 \leqslant s \leqslant k, G_{0} \subset \subset$ $G_{h} \subset \subset G_{1} \subset \subset G$. Then, for any $\eta \in S_{k, r}^{h}\left(\Omega_{1}\right)$,

$$
\begin{aligned}
\|e\|_{s, G_{0}} & \leqslant\|u-\eta\|_{s, G}+\left\|\eta-u_{h}\right\|_{s, G_{h}} \leqslant\|u-\eta\|_{s, G}+h^{1-s}\left\|\eta-u_{h}\right\|_{1, G_{h}} \\
& \leqslant\|u-\eta\|_{s, G}+h^{1-s}\|u-\eta\|_{1, G}+h^{1-s}\|e\|_{1, G},
\end{aligned}
$$

where we used (2.4). We now choose $\eta$ satisfying (2.1) and estimate $\|e\|_{1}, G_{1}$ using (5.26) with $A_{e}=0$ and $G_{1}$ in place of $G_{0}$. We obtain

$$
\|e\|_{s, G_{0}} \leqslant C\left(h^{l-s}\|u\|_{l, G}+h^{1-s}\|e\|_{p, G}\right),
$$

which completes the proof.

Proofs of Theorems 5.1 and 5.2. Let $d=\min \left(d_{0} / 2, d_{1} / 2\right)$ where $d_{0}=$ $\operatorname{dist}\left(\bar{\Omega}_{0}, \partial \Omega_{1}\right)$. Cover $\bar{\Omega}_{0}$ with a finite number of spheres $G_{0}\left(x_{i}\right), i=1, \cdots, m$, centered at $x_{i} \in \bar{\Omega}_{0}$ with $\operatorname{diam} G_{0}\left(x_{i}\right)=d$. Let $G\left(x_{i}\right), i=1, \cdots, m$, be corresponding concentric spheres with $\operatorname{diam} G\left(x_{i}\right)=2 d$. Applying (5.26) and (5.28) we have for $s=0,1$ and $1 \leqslant l \leqslant r$, 


$$
\begin{aligned}
\|e\|_{s, G_{0}\left(x_{i}\right)} & \leqslant C_{i}\left(h^{l-s}\|u\|_{l, G\left(x_{i}\right)}+\|e\|_{p, G\left(x_{i}\right)}\right) \\
& \leqslant C_{i}\left(h^{l-s}\|u\|_{l, \Omega_{1}}+\| e L_{p, \Omega_{1}}\right)
\end{aligned}
$$

and from (5.29) for $2 \leqslant s \leqslant k$ and $s \leqslant l \leqslant r$,

$$
\|e\|_{s, G_{0}\left(x_{i}\right)} \leqslant C_{i}\left(h^{l-s}\|u\|_{l, \Omega_{1}}+h^{1-s}\|e\|_{p, \Omega_{1}}\right) .
$$

The inequalities (5.1) and (5.2) follow from (5.30) and (5.31), which concludes the proof of Theorem 5.1. The proof of Theorem 5.2 follows precisely in the same way from the inequalities (5.26) and (5.28) with the help of (3.6). We shall leave the details to the reader.

6. Convergence of Difference Quotients. The estimates for the error and its derivatives given in the previous section are valid, for example, for subspaces $S_{k, r}^{h}$ which may be defined on nonuniform meshes. In this section, we shall consider subspaces which have certain translation invariant properties (which are satisfied, for example, by spline subspaces on a uniform mesh). This will allow us to obtain some results concerning the rate of convergence of difference quotients of the approximation $u_{h}$ to derivatives of $u$.

Let $\mu=\left(\mu_{1}, \cdots, \mu_{N}\right)$ be a multi-integer. We define the translation operator

$$
T_{h}^{\mu} v(x)=v(x+\mu h)
$$

and the forward difference quotients

$$
\partial_{h, j} v=h^{-1}\left(T_{h}^{e} j-I\right) u,
$$

where $I$ is the identity operator and, for any multi-index $\alpha$,

$$
\partial_{h}^{\alpha} u \equiv \partial^{\alpha} u=\partial_{h, 1}^{\alpha_{1}} \cdots \partial_{h, N}^{\alpha} u .
$$

We consider difference operators $Q_{h}$ of order $m$ of the form

$$
Q_{h} u=\sum_{\nu,|\beta| \leqslant m} C_{\nu \beta} T_{h}^{\nu} \partial^{\beta} u,
$$

where the $C_{\nu \beta}$ are the constants and all but a finite number of the $C_{\nu \beta}$ vanish. We note that $Q_{h} u$ may be written as a linear combination of translation operators with coefficients which depend only on $h$.

In what follows, we shall make use of the discrete Leibnitz rule

$$
\partial_{h}^{\alpha}(u v)=\sum_{\beta \leqslant \alpha}\left(\begin{array}{l}
\alpha \\
\beta
\end{array}\right) T_{h}^{\beta} \partial_{h}^{\alpha-\beta} u \partial_{h}^{\beta} v, \quad\left(\begin{array}{l}
\alpha \\
\beta
\end{array}\right)=\left(\begin{array}{l}
\alpha_{1} \\
\beta_{1}
\end{array}\right) \cdots\left(\begin{array}{l}
\alpha_{N} \\
\beta_{N}
\end{array}\right) .
$$

Our additional assumption on the subspaces $S_{k, r}^{h}(\Omega)$ is as follows:

A.4. Let $\nu$ be any multi-integer, fixed but arbitrary, and let $\Omega_{0} \subset \subset \Omega_{1}$, then there exists an $h_{1}$ (in general depending on $\nu, \Omega_{0}$ and $\Omega_{1}$ ) such that for all $h \in\left(0, h_{1}\right]$

$$
T_{h}^{\nu} \varphi \in \stackrel{\circ}{S}_{k, r}^{h}\left(\Omega_{1}\right) \quad \forall \varphi \in \stackrel{\circ}{S}_{k, r}^{h}\left(\Omega_{0}\right) .
$$


We shall now prove

THEOREM 6.1. Suppose that the conditions of Theorem 5.1 are satisfied and in addition A.4 holds. Let $Q_{n}$ be a finite difference operator of order $m$ of the form (6.1). If $1 \leqslant l \leqslant r, u \in H^{m+l}\left(\Omega_{1}\right)$ and $p$ is any nonnegative integer; fixed but arbitrary, then there exists an $h_{0}$ such that for all $h \in\left(0, h_{0}\right]$

$$
\left\|Q_{h} e\right\|_{0, \Omega_{0}} \leqslant C\left(h^{l}\|u\|_{l+m, \Omega_{1}}+\|e\|_{p, \Omega_{1}}\right) \text {, }
$$

where $C=C\left(p, \Omega_{0}, \Omega_{1}, a_{i j}, b_{i}, c\right)$.

Proof. Let us first remark that the proof in the case that $B(u, v)$ has constant coefficients is almost an immediate consequence of Theorem 5.1 and A.4. For, if $\Omega_{0} \subset \subset \Omega_{0}^{\prime} \subset \subset \Omega_{1}$, then it is easily seen that for $h$ sufficiently small

$$
B\left(Q_{h} e, \varphi\right)=B\left(e, Q_{h}^{*} \varphi\right)=0 \quad \forall \varphi \in \stackrel{\circ}{S}_{k, r}^{h}\left(\Omega_{0}^{\prime}\right) .
$$

Here, $Q_{h}^{*}$ is the difference operator adjoint to $Q_{h}$. Noticing that for $p^{\prime}=m+p$, $\left\|Q_{h} e\right\|_{-p^{\prime}, \Omega_{0}^{\prime}} \leqslant C\|e\|_{-p, \Omega_{0}}$, we may apply Theorem 5.1, with $\Omega_{0}, \Omega_{0}^{\prime}$ and $p^{\prime}$ replacing $\Omega_{0}, \Omega_{1}$ and $p$, respectively, and (6.3) follows immediately.

For the case of variable coefficients, we first note that any difference operator of the form (6.2) is a linear combination of products of translations and difference quotients which commute. It is obviously sufficient to prove $(6.3)$ in the case that $Q_{h} e=T_{h}^{\nu} \partial^{\alpha} e$. We shall first show that

$$
\left\|T_{h}^{\nu} \partial^{\alpha} e\right\|_{1, \Omega_{0}} \leqslant C\left(h^{l-1}\|u\|_{l+|\alpha|, \Omega_{1}}+\|e\|_{p, \Omega_{1}}\right) .
$$

Our proof will proceed by induction. Since

$$
\left\|T_{h}^{\nu} e\right\|_{1, \Omega_{0}} \leqslant\|e\|_{1, \Omega_{0}^{\prime}}
$$

for $h$ sufficiently small, the inequality (6.5), in the case $|\alpha|=0$, follows immediately from (6.6) and (5.1).

Let us now assume that $|\alpha| \geqslant 1$. In view of the inequality (6.6), we may restrict ourselves to estimating $\partial^{\alpha} e$. Let us investigate the equation satisfied by $\partial^{\alpha} e$. For ease of computation, we shall assume that $B(u, v)$ is of the form

$$
B(u, v)=\int_{\Omega_{1}} \sum_{i, j=1}^{N} a_{i j} D_{i} u D_{j} v d x
$$

the lower order terms can be handled in exactly the same manner. Using the Leibnitz rule, we have

$$
\begin{aligned}
B\left(\partial^{\alpha} e, \varphi\right)= & \int_{\Omega_{1}} \sum_{i, j=1}^{N} \partial^{\alpha}\left(a_{i j} D_{i} e\right) D_{j} \varphi d x \\
& -\int_{\Omega_{1}} \sum_{i, j=1}^{N} \sum_{\beta<\alpha}\left(\begin{array}{c}
\alpha \\
\alpha-\beta
\end{array}\right) \partial^{\alpha-\beta} a_{i j} T_{h}^{\alpha-\beta} \partial^{\beta} D_{i} e D_{j} \varphi d x .
\end{aligned}
$$

It follows, in a manner similar to that used in proving (6.4), that, for $h$ sufficiently small, the first integral on the right-hand side of (6.7) vanishes for all $\varphi \in \stackrel{\circ}{S}_{k, r}^{h}\left(\Omega_{0}^{\prime}\right)$. 
Therefore, for $|\alpha|=t+1$,

(6.8) $B\left(\partial^{\alpha} e, \varphi\right)=A_{e}(\varphi)=-\int_{\Omega_{1}} \sum_{i, j=1}^{N} \sum_{\beta<\alpha}\left(\begin{array}{c}\alpha \\ \alpha-\beta\end{array}\right) \partial^{\alpha-\beta} a_{i j} T_{h}^{\alpha-\beta} \partial^{\beta} D_{i} e D_{j} \varphi d x$

where $|\beta| \leqslant t$. We may therefore apply Theorem 5.2 with $\partial^{\alpha} e$ replacing $e$, and $\Omega_{0}$ and $\Omega_{0}^{\prime}$ replacing $\Omega_{1}$. For $h$ sufficiently small and $\Omega_{0} \subset \subset \Omega_{0}^{\prime} \subset \subset \Omega_{0}^{\prime \prime} \subset \subset$ $\Omega_{1}$, we have

$$
\left\|A_{e}\right\|_{1, \Omega_{0}^{\prime}} \leqslant C \sum_{|\beta| \leqslant t}\left\|\partial^{\beta} e\right\|_{1, \Omega_{0}^{\prime \prime}}
$$

By our induction hypothesis, we have for each $|\beta| \leqslant t$

$$
\left\|\partial^{\beta} e\right\|_{1, \Omega_{0}^{\prime \prime}} \leqslant C\left(h^{l-1}\|u\|_{l+t, \Omega_{1}}+\|e\|_{p, \Omega_{1}}\right) .
$$

The inequality (6.5) now easily follows from (5.6), (6.9) and (6.10).

The proof of (6.3) now follows from (6.4) and (5.5) in the same manner. One only has to observe that, in place of (6.9), one has the inequality

$$
\left\|A_{e}\right\|_{0, \Omega_{0}^{\prime}} \leqslant C \sum_{\beta<\alpha}\left\|\partial^{\beta} e\right\|_{0}, \Omega_{0}^{\prime \prime}
$$

The details will be left to the reader.

Our next concern is the convergence of difference quotients of $u_{h}$ to derivatives of $u$. We shall say that a difference operator $Q_{h}=Q_{h}^{\alpha}$ of order $|\alpha|$ approximates a derivative $D^{\alpha}$ with order of accuracy $t$ in $L_{2}$, if for any pair of domains $\Omega_{0} \subset \subset$ $\Omega_{1}$

$$
\left\|D^{\alpha} u-Q_{h}^{\alpha} u\right\|_{0, \Omega_{0}} \leqslant C\left(\Omega_{0}, \Omega_{1}\right) h^{t}\|u\|_{t+|\alpha|, \Omega_{1}},
$$

for all $h$ sufficiently small and $u \in H^{t+|\alpha|}\left(\Omega_{1}\right)$.

THEOREM 6.2. Suppose that the conditions of Theorem 6.1 are satisfied and let $Q_{h}^{\alpha}$ approximate $D^{\alpha}$ with order of accuracy $r$ in $L_{2}$. Furthermore, let $p$ be a nonnegative integer, fixed but arbitrary. There exists an $h_{1}>0$ such that for all $h \in\left(0, h_{1}\right]$

$$
\left\|D^{\alpha} u-Q_{h}^{\alpha} u_{h}\right\|_{0, \Omega_{0}} \leqslant C\left(h^{r}\|u\|_{r+|\alpha|, \Omega_{1}}+\|e\|_{p, \Omega_{1}}\right)
$$

where $C=C\left(p, \Omega_{0}, \Omega_{1}, a_{i j}, b_{i}, c\right)$.

We remark that, in contrast to the results of Theorem 5.1, Theorem 6.2 says that if $u$ is sufficiently smooth on $\Omega_{1}$ and $Q_{n}^{\alpha}$ a sufficiently good approximation to $D^{\alpha}$, then the rate of convergence of $Q_{h}^{\alpha} u_{h}$ to $D^{\alpha}$ is of order $h^{r}$ plus the term $\|e\|_{-p, \Omega_{1}}$.

Proof. The proof is obvious because of the inequality

$$
\left\|D^{\alpha} u-Q_{h}^{\alpha} u_{h}\right\|_{0, \Omega_{0}} \leqslant\left\|D^{\alpha} u-Q_{h}^{\alpha} u\right\|_{0, \Omega_{0}}+\left\|Q_{h}^{\alpha} e\right\|_{0, \Omega_{0}} .
$$


7. Examples. In this section, we shall exemplify the theory given in the previous sections by considering specific methods for approximating specific elliptic boundary value problems on a bounded domain $\Omega \subset \subset \mathbf{R}^{N}$. In what follows, we shall assume that the subspaces $S_{k, r}^{h}(\Omega) \subset H^{k}(\Omega), k \geqslant 1$, used in approximating the solution, are such that their restrictions to a given subdomain $\Omega_{1} \subset \subset \Omega$ satisfies the assumptions $A_{1}, A_{2}$ and $A_{3}$. Several examples of such subspaces have been given in Section 2.

For the purposes of our applications, we shall further assume that $S_{k, r}^{h}(\Omega)$ has the following approximation property on $\Omega$ : There exists a constant $C$ independent of $u$ and $h$ such that for $k \leqslant t \leqslant r$ and all $u \in H^{t}(\Omega)$

$$
\inf _{h} \in S_{k, r}^{h}(\Omega) \sum_{j=1}^{k} h^{j}\left\|u-u_{h}\right\|_{j, \Omega} \leqslant C h^{t}\|u\|_{t, \Omega} .
$$

We shall sometimes use subspaces $S_{k, r}^{h}(\Omega)$ whose elements are required to vanish on $\partial \Omega$. In this case, we shall require that (7.1) hold only for $u \in H^{t}(\Omega) \cap \stackrel{\circ}{H}^{1}(\Omega)$. The assumption (7.1) holds for the examples cited in Section 2, provided for instance $\Omega$ is a Lipschitz domain. If additional requirements on the subspaces are needed when considering a specific method, we shall indicate this at that time.

Example 1. The Neumann Problem. Let $\Omega$ be a bounded domain in $\mathbf{R}^{N}$ with smooth boundary $\partial \Omega$. Let $u$ be a solution of

$$
\begin{aligned}
-\Delta u+u=f & \text { in } \Omega, \\
\partial u / \partial n=0 & \text { on } \partial \Omega,
\end{aligned}
$$

where $\partial u / \partial n$ is the outward normal derivative of $u$ to $\partial \Omega$. Let $u_{h} \in S_{k, r}^{h}(\Omega)$ be the approximate solution defined by

$$
B\left(u_{h}, \varphi\right)=\int_{\Omega} \sum_{i=1}^{N} \frac{\partial u_{h}}{\partial x_{i}} \frac{\partial \varphi}{\partial x_{i}} d x+\int_{\Omega} u_{h} \varphi d x=\int_{\Omega} f \varphi d x=B(u, \varphi)
$$

for all $\varphi \in S_{k, r}^{h}(\Omega)$. Here $S_{k, r}^{h}(\Omega)$ need not satisfy any conditions on $\partial \Omega$ and any one of the previous examples of subspaces can be used. We note that this procedure is well defined if $f \in H^{-1}(\Omega)$ and that in general

$$
\begin{aligned}
\|u\|_{1, \Omega} & \leqslant C\|f\|_{1}, \Omega, \\
\|u\|_{s+2, \Omega} & \leqslant C(s)\|f\|_{s, \Omega} \text { if } 0 \leqslant s .
\end{aligned}
$$

It was shown in [7], that, if (7.1) holds, then for $e=u-u_{h}$

$$
\|e\|_{2-r, \Omega} \leqslant\|e\|_{2-r, \Omega} \leqslant C h^{r-2+t}\|u\|_{t, \Omega}, \quad 1 \leqslant t \leqslant r, r \geqslant 1 .
$$

If $\Omega_{0} \subset \subset \Omega_{1} \subset \subset \Omega$, then our interior Eqs. (2.1) are satisfied for $h$ sufficiently small and, using the inequality $\|e\|_{2-r, \Omega_{1}} \leqslant\|e\|_{2-r, \Omega}$, we obtain from Theorem 
5.1 that, if $u \in H^{r}\left(\Omega_{1}\right) \cap H^{2}(\Omega)$, then

$$
\|e\|_{0, \Omega_{0}} \leqslant C h^{r}\left(\|u\|_{r, \Omega_{1}}+\|u\|_{2, \Omega}\right) .
$$

If $u \in H^{r}\left(\Omega_{1}\right) \cap H^{1}(\Omega)$, then

$$
\|e\|_{1, \Omega_{0}} \leqslant C h^{r-1}\left(\|u\|_{r, \Omega_{1}}+\|u\|_{1, \Omega}\right) \text {. }
$$

We note that the best estimates one can obtain on all of $\Omega$ for any $u \in H^{r}(\Omega)$ is

$$
\|e\|_{0, \Omega} \leqslant C h^{r}\|u\|_{r, \Omega}, . \quad\|e\|_{1, \Omega} \leqslant C h^{r-1}\|u\|_{r, \Omega} .
$$

In contrast to those, (7.6) and (7.7) say that we obtain quasi-optimal estimates in $L_{2}\left(\Omega_{0}\right)$ and $H^{1}\left(\Omega_{0}\right)$ provided in both cases $u \in H^{r}\left(\Omega_{1}\right)$ and $u \in H^{2}(\Omega)$ and $H^{1}(\Omega)$, respectively. It follows from interior a priori estimates for elliptic equations that (7.6) and (7.7) may be replaced by

$$
\|e\|_{0, \Omega_{0}} \leqslant C h^{r}\left(\|f\|_{r-2, \Omega_{1}}+\|f\|_{0, \Omega}\right)
$$

and

$$
\|e\|_{1, \Omega_{0}} \leqslant C h^{r-1}\left(\|f\|_{r-2, \Omega_{1}}+\|f f\|_{-1, \Omega}\right)
$$

respectively.

In this particular problem, we are at liberty to choose our subspaces to be defined on a uniform mesh; hence, the results of Section 6 on difference quotients are applicable.

If $\alpha$ is any multi-index and $Q_{h}^{\alpha}$ is a finite difference operator of the form (6.1) which approximates $D^{\alpha}$ with order of accuracy $r$, then, for $h$ sufficiently small, we obtain from Theorem 6.2 that

$$
\left\|D^{\alpha} u-Q_{h}^{\alpha} u_{h}\right\|_{0, \Omega_{0}} \leqslant C h^{r}\left(\|u\|_{r+|\alpha|, \Omega_{1}}+\|u\|_{2, \Omega}\right) .
$$

One can show that in terms of data

$$
\left\|D^{\alpha} u-Q_{h}^{\alpha} u_{h}\right\|_{0, \Omega_{0}} \leqslant C h^{r}\left(\|f\|_{r+|\alpha|-2, \Omega_{1}}+\|f\|_{0, \Omega}\right) .
$$

Example 2. The Dirichlet Problem, Babuska's Method of Lagrange Multipliers and Two Methods of Nitsche. In Babuska [3] and Nitsche [12] and [13], methods are introduced for approximating solutions of

$$
\begin{gathered}
-\Delta u=f \text { in } \Omega, \\
u=0 \text { on } \partial \Omega
\end{gathered}
$$

(in [3] and [12] in general $u=g$ on $\partial \Omega$ ) in which the approximating subspace need not satisfy boundary conditions. In [3], the subspaces $S_{k, r}^{h}(\Omega)$ need not satisfy any additional requirements and may be taken to be any one of the examples given. However, another one-parameter family of subspaces is introduced, which are defined only on $\partial \Omega$ and which may be thought of as approximating the normal 
derivative of $u$ on $\partial \Omega$. In [12] and [13], subspaces are constructed which have certain additional properties near $\partial \Omega$. What is important here is that subspaces used in these methods can be chosen to satisfy the conditions of Theorem 5.1 and, if $\Omega_{0} \subset \subset \Omega_{1} \subset \subset \Omega$ and $h$ is sufficiently small, then the interior equations for all of these methods are the same, i.e., of the form

$$
B\left(u_{h}, \varphi\right)=\int_{\Omega} \sum_{i=1}^{N} \frac{\partial u_{h}}{\partial x_{i}} \frac{\partial \varphi}{\partial x_{i}} d x=(f, \varphi)=B(u, \varphi) \quad \forall \varphi \in \stackrel{\circ}{S}_{k, r}^{h}\left(\Omega_{1}\right)
$$

It was shown in [7] that the estimate (7.5) is valid for all three of these methods provided $\partial \Omega$ is smooth and hence it follows that the error estimates (7.6), (7.7) and (if a uniform mesh is used on $\Omega_{1}$ ) (7.10) remain valid. We remark that the same estimates hold if in (7.12) the Laplacian is replaced by any second order elliptic operator of the form (1.4), such that the corresponding boundary value problem has a unique solution.

Example 3. Dirichlet's Problem on the Unit Square. Let us again consider the problem (7.12), (7.13) on $\Omega$ in $\mathbf{R}^{2}$ where $\Omega=\left\{x \mid 0<x_{i}<1, i=1,2\right\}$. We shall approximate $u$ using subspaces $S_{k, r}^{h}(\Omega)$ which vanish on $\partial \Omega$. Let $u_{h} \in$ $S_{k, r}^{h}(\Omega)$ be the approximate solution defined by

$$
B\left(u_{h}, \varphi\right)=\int_{\Omega} \sum_{i=1}^{2} \frac{\partial u_{n}}{\partial x_{i}} \frac{\partial \varphi}{\partial x_{i}} d x=(f, \varphi)=B(u, \varphi) \quad \forall \varphi \in S_{k, r}^{h} .
$$

In order to obtain interior estimates, we estimate $\|e\|_{2-r,}$. We shall show that the estimate (7.5) remains valid here. The basic idea in obtaining this estimate in this case may be found in [18]. For completeness, we shall outline the proof. Now

$$
\|e\|_{2-r, \Omega}=\sup _{v \in C_{0}^{\infty}(\Omega)}(e, v) /\|v\|_{r-2, \Omega}
$$

For each $v \in C_{0}^{\infty}(\Omega)$, let $\psi$ be the solution of (7.12), (7.13) with $f=v$. In general, solutions of (7.12), (7.13) need not be smooth even for smooth $f$ and the best one can say is that, if $f \in C^{\infty}(\bar{\Omega})$, then $u \in H^{3-\epsilon}(\Omega) \cap \stackrel{\circ}{H}^{1}(\Omega)$ for any $\epsilon>0$. However, in the case that $v \in C_{0}^{\infty}(\Omega)$, then, in fact, $\psi \in C^{\infty}(\bar{\Omega})$ and the a priori estimate

$$
\|\psi\|_{s, r} \leqslant C\|v\|_{s-2, \Omega}, \quad 0 \leqslant s,
$$

holds, where $C$ is independent of $v$ and depends only on $s$. In order to see this, we notice that $\psi$ may be continued to a square domain $G$ where $\bar{\Omega} \subset G$ as a solution of $-\Delta \psi=g \quad$ in $G, \psi=0$ on $\partial G$ where $g \in C_{0}^{\infty}(G), g=v$ on $\bar{\Omega}$. This is done by repeated reflection of $\psi$ across the edges as an odd function. It then follows from standard interior estimates for solutions of (7.12) that $\psi$ is $C^{\infty}$ on every subdomain of $G$ and hence on $\bar{\Omega}$. The estimate (7.16) is valid for 
smooth solutions. Hence, for any $\eta \in S_{k, r}^{h}$, we have, using (7.14), that $(e, v)=$ $-(e, \Delta \psi)=B(e, \psi)=B(e, \psi-\eta)$ or

$$
|(e, v)| \leqslant\|e\|_{1, \Omega} \inf _{\eta \in S_{k, r}^{h}}\|\psi-\eta\|_{1, \Omega} \leqslant C h^{r-1}\|e\|_{1, \Omega}\|\psi\|_{r, \Omega} .
$$

In view of (7.17), (7.16), (7.15) and the fact that $u_{h}$ is the best approximation in $S_{k, r}^{h}$ to $u$ in $\stackrel{\circ}{H}^{1}(\Omega)$, the inequality (7.5) now follows. This immediately implies that the estimates (7.10), (7.6) and (7.7) are valid in this case, even though there are corners. However, the method of proof of the estimate (7.4) depends very much on the fact that the interior angles of $\Omega$ are $\pi / 2$. We shall now treat the case of the $L$-shaped membrane where we are generally not able to show that (7.5) holds.

Example 4. Dirichlet's Problem on the L-shaped Membrane. We again consider

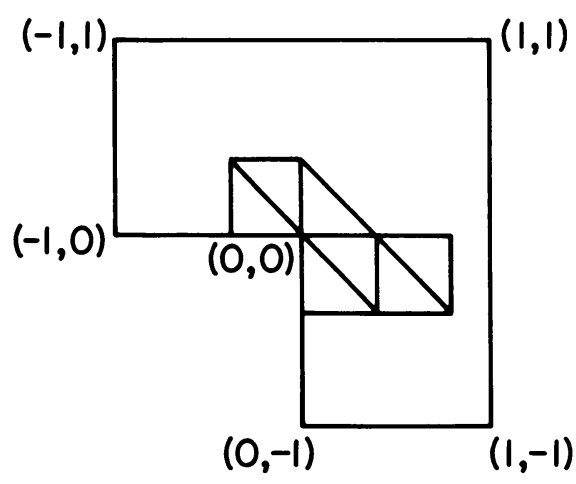
(7.12) and (7.13) where $\Omega$ is the $L$-shaped domain (see figure). For our subspace, we choose $S_{k, r}^{h}(\Omega)=S_{1,2}^{h}$ to be piecewise linear functions on a uniform triangulation of $\Omega$ which vanish on $\partial \Omega$ and again denote by $u_{h}$ the approximate solution determined by (7.14). Now, in general, if $f$ is $C^{\infty}(\bar{\Omega})$ or even $C_{0}^{\infty}(\Omega)$, then the most one can say of solutions $u$ of (7.12), (7.13) is that $u \in$ following estimate (cf. [9] ) is valid for any $f \in H^{-1 / 3-\epsilon}(\Omega)$,

$$
\|u\|_{5 / 3-\epsilon, \Omega} \leqslant C\|f\|_{-1 / 3-\epsilon, \Omega} .
$$

We shall now estimate $\|e\|_{-p, \Omega}$. For any $p \geqslant 0$, we have

$$
\|e\|_{-p, \Omega}=\sup _{v \in C_{0}^{\infty}}(e, v) /\|v\|_{p, \Omega} .
$$

Again, let $\Delta \psi=v$ on $\Omega, \psi=0$ on $\partial \Omega$; then, in general, $\psi \in H^{5 / 3-\epsilon}(\Omega)$ for any $\epsilon>0$ (in contrast to the unit square where $\psi \in C^{\infty}(\bar{\Omega})$ ). We then have for any $\eta \in S_{1,2}^{h}(\Omega)$

$$
\begin{aligned}
(e, v) & =-(e, \Delta \psi)=B(e, \psi)=B(e, \psi-\eta) \leqslant\|e\|_{1, \Omega} \inf _{\eta \in S_{1,2}^{h}(\Omega)}\|\psi-\eta\|_{1, \Omega} \\
& \leqslant C h^{4 / 3-2 \epsilon}\|u\|_{5 / 3-\epsilon, \Omega}\|\psi\|_{5 / 3-\epsilon, \Omega} .
\end{aligned}
$$

From this it follows that for any $p>0$

$$
\|e\|_{-p, \Omega} \leqslant C(\epsilon) h^{4 / 3-2 \epsilon}\|u\|_{5 / 3-\epsilon, \Omega} .
$$

Hence, in general, the convergence seems to be no better in any negative norm than 
in $L_{2}(\Omega)$. In particular, for all $u \in H^{5 / 3-\epsilon}$, we have

$$
\|e\|_{0, \Omega} \leqslant C h^{4 / 3-2 \epsilon}\|u\|_{5 / 3-\epsilon, \Omega},
$$

where $C$ is independent of $h$ and $u$.

If we apply the estimate (7.18) to the results of Theorem 5.1, we see that for all $u \in H^{2}\left(\Omega_{1}\right)$ (here $r=2$ )

$$
\|e\|_{0, \Omega_{0}} \leqslant C\left(h^{2}\|u\|_{2, \Omega_{1}}+h^{4 / 3-2 \epsilon}\|u\|_{5 / 3-\epsilon, \Omega}\right) .
$$

Hence, for $h$ small, the order of convergence is $h^{4 / 3-2 \epsilon}$ which is no better than that over all of $\Omega$ and the estimate (7.20) yields nothing new since (7.18) is also trivially valid with $\|e\|_{0, \Omega}$ replaced by $\|e\|_{0, \Omega_{0}}$.

In some sense, this inequality is sharp. Following an idea of Babußka, one can show that, for each $h$, there exists a function $u=U^{h}$ such that

$$
\|e\|_{0, \Omega_{0}}=\left\|U^{h}-u_{h}\right\|_{0, \Omega_{0}} \geqslant C h^{4 / 3-\epsilon}\left\|U^{h}\right\|_{5 / 3-\epsilon, \Omega} .
$$

We shall not give the details here.

However, we note that this does not say that for a given fixed $u$ the rate of convergence in the interior is not higher than $n^{4 / 3-\epsilon}$ This is an open question at this time.

Institut fur Angewandte Mathematik

Universität Freiburg

Freiburg, Germany

Department of Mathematics

Cornell University

Ithaca, New York 14850

1. S. AGMON, Lectures on Elliptic Boundary Value Problems, Van Nostrand Math. Studies, no. 2, Van Nostrand, Princeton, N. J., 1965. MR 31 \#2504.

2. J. P. AUBIN, “Approximation des problèmes aux limites non homogènes et régularité de la convergence," Calcolo, v. 6, 1969, pp. 117-139.

3. I. BABUŠKA, The Finite Element Method with Lagrangian Multipliers, Technical Note BN-724, Institute for Fluid Dynamics and Appl. Math., University of Maryland, College Park, Md., 1972.

4. I. BABUŠKA, Numerical Solution of Boundary Value Problems by the Perturbed Variational Principle, Technical Note BN-624, University of Maryland, College Park, Md., 1969.

5. JU. M. BEREZANSKII, Expansions in Eigenfunctions of Selfadjoint Operators, "Naukova Dumka", Kiev, 1965 ; English transl., Transl. Math. Monographs, vol. 17, Amer. Math. Soc., Providence, R. I., 1968. MR 36 \#5768; \#5769.

6. J. H. BRAMBLE \& S. HILBERT, "Bounds for a class of linear functionals with applications to Hermite interpolation," Numer. Math., v. 16, 1971, pp. 362-369. MR 44 \#7704.

7. J. H. BRAMBLE \& J. E. OSBORN, "Rate of convergence estimates for non-selfadjoint eigenvalue approximations," Math. Comp., v. 27, 1973, pp. 525-550. 
8. J. H. BRAMBLE \& M. ZLÁMAL, "Triangular elements in the finite element method," Math. Comp., v. 24, 1970, pp. 809-820. MR $43 \# 8250$.

9. R. B. KELLOGG, "Higher order singularities for interface problems," Proceedings of Conference on The Mathematical Foundations of the Finite Element Method with Applications to Partial Differential Equations (edited by A. K. Aziz), Academic Press, New York, 1972, pp. 589-602.

10. R. B. KELLOGG, Interpolation Between Subspaces of a Hilbert Space, Technical Note BN-719, Institute for Fluid Dynamics and Appl. Math., University of Maryland, College Park, Md., 1972.

11. J. L. LIONS \& E. MAGENES,'Problème aux limites non homogènes et applications, Vol. 1, Travaux et Recherches Mathématiques, no. 17, Dunod, Paris, 1968. MR 40 \#512.

12. J. A. NITSCHE, “Über ein Variationsprinzip zur Lösung von Dirichlet Problemen bei Verwendung von Teilräumen, die keinen Randbedingungen unterworfen sind," Abh. Math. Sem. Univ. Hamburg, v. 36, 1971 , pp. 9-15.

13. J. A. NITSCHE, "A projection method for Dirichlet-problems using subspaces with nearly zero boundary conditions," Proceedings of Conference on The Mathematical Foundations of the Finite Element Method with Applications to Partial Differential Equations (edited by A. K. Aziz), Academic Press, New York, 1972, pp. 603-627.

14. J. A. NITSCHE, "Umkehrsätze für Spline Approximationen," Compositio Math., v. 21, 1969, pp. 400-416. MR 41 \#4074.

15. J. A. NITSCHE, "Ein Kriterium für die Quasi-Optimalität des Ritzschen Verfahrens," Numer. Math., v. 11, 1968, pp. 346-348. MR 38 \#1823.

16. J. A. NITSCHE, "Interior error estimates for projection methods," Proceedings of the Czechoslovak Conference on Differential Equations and Their Applications, Brno 1972.

17. J. A. NITSCHE \& A. H. SCHATZ, "On local approximation properties of $L_{2}$ projections on spline subspaces," Applicable Anal., v. 2, 1972, pp. 161-168.

18. L. SERBIN, A Computational Investigation of Least Squares and Other Projection Methods for the Approximate Solution of Boundary Value Problems, Doctoral Thesis, Cornell University, Ithaca, N. Y., 1971.

19. I. J. SCHOENBERG, Approximation with Special Emphasis on Spline Functions, Academic Press, New York, 1969. MR 40 \#4638.

20. V. THOMEE \& B. WESTERGREN, "Elliptic difference equations and interior regularity," Numer. Math., v. 11, 1968, pp. 196-210. MR 36 \#347.

21. V. THOMÉE, "Discrete interior Schauder estimates for elliptic difference operators," SIAM J. Numer. Anal., v. 5, 1968, pp. 626-645. MR 38 \#6781.

22. M. ZLÁMAL, “A Finite element procedure of second order accuracy," Numer. Math., v. 14, 1969/70, pp. 394-402. MR 41 \#1233. 